\title{
A Mobile Scanner for Probing Liquid Samples in Everyday Settings
}

Weiwei Jiang

The University of Tokyo

Bunkyo-ku, Tokyo, Japan

wjiang@akg.t.u-tokyo.ac.jp

Gabriele Marini

Niels van Berkel

Zhanna Sarsenbayeva

Chu Luo

The University of Melbourne

Melbourne, VIC, Australia

\{firstname.lastname\}

@student.unimelb.edu.au

\section{Xin $\mathrm{He}$}

Anhui Normal University

Wuhu, Anhui, China

xin.he@ahnu.edu.cn
Tilman Dingler

The University of Melbourne

Melbourne, VIC, Australia

tilman.dingler@unimelb.edu.au

Yoshihiro Kawahara

The University of Tokyo

Bunkyo-ku, Tokyo, Japan

kawahara@akg.t.u-tokyo.ac.jp

Vassilis Kostakos

The University of Melbourne

Melbourne, VIC, Australia

vassilis.kostakos@unimelb.edu.au

Permission to make digital or hard copies of all or part of this work for personal or classroom use is granted without fee provided that copies are not made or distributed for profit or commercial advantage and that copies bear this notice and the full citation on the first page. Copyrights for components of this work owned by others than the author(s) must be honored. Abstracting with credit is permitted. To copy otherwise, or republish, to post on servers or to redistribute to lists, requires prior specific permission and/or a fee. Request permissions from permissions@acm.org. Copyright held by the owner/author(s). Publication rights licensed to ACM. UbiComp/ISWC'18 Adjunct,, October 8-12, 2018, Singapore, Singapore ACM 978-1-4503-5966-5/18/10.

\begin{abstract}
Our work investigates the use of a Near InfraRed Spectroscopy scanner for the identification of liquids. While previous work has shown promising results for the identification of solid objects, identifying liquids poses additional challenges. These challenges include light scattering and low reflectance caused by the transparency of liquids, which interfere with the infrared measurement. We develop a prototype solution consisting of a 3D printed clamp that attaches to a tube, such that it blocks ambient light from interfering. Our preliminary results indicate that our prototype works, and we demonstrate this by measuring sugar levels in a liquid solution.
\end{abstract}

\section{Author Keywords}

NIRS; liquids; sugar; light scattering; 3D printing.

\section{ACM Classification Keywords}

H.5.m [Information interfaces and presentation (e.g., $\mathrm{HCl}$ )]: Miscellaneous

\section{Introduction}

Given the recent advances in deep learning, computer vision can outperform humans in several object identification tasks [3]. However, previous research focuses mostly on solid-state objects identification, whereas the identification of liquids remains under-explored. Vision-based approaches to 


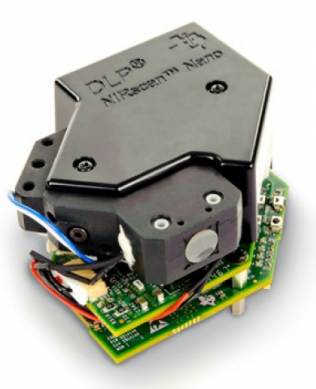

Figure 1: Texas Instruments DLP NIRscan EVM. identify liquids are likely to be imprecise, due to the lack of physical form of liquids, and their generic color which can often be affected by ambient light.

One promising direction for liquid identification is through the analysis of the liquid's chemical composition. Obtaining chemical composition information of an object readily and rapidly, however, is in itself another challenge [1]. In this study, we explore the possibility of using a recently released miniaturized Near InfraRed Spectroscopy (NIRS) scanner. The device emits Near InfraRed (NIR) light that penetrates the surface of samples and is reflected or absorbed by the object's molecules. The reflected light is then captured and forms a spectrum with respect to the chemical composition, resulting in a chemical fingerprint that can be used to identify objects, including liquids.

Conventionally, NIRS requires a dedicated device operated by professionals. However, current miniaturized NIRS scanners offer the possibility for non-professionals to access this technique in a ubiquitous context [7]. Previous work has shown great examples of non-professionals utilizing this device [10]. Specifically, Klakegg et al. have demonstrated that nurses could use this technique to accurately identify pills [9]. Nevertheless, their work focused on identification of solid objects in daily life, meanwhile the identification of non-solid objects, specifically liquids, remains an open challenge.

In this paper, we present a prototype that extends the capabilities of a miniaturized NIRS scanner to identify liquids. The scanner (Texas Instruments DLP NIRscan EVM [7] as shown in Figure 1) weighs 80 grams and costs less than 1000 USD for an evaluation kit. We expect this device to become cheaper following mass production. To demonstrate our prototype's capabilities in identifying liquids, we conducted an experiment with solutions of different sugar concentrations (common sucrose). Our results show the feasibility of our prototype being used for analyzing sugar levels in a solution. We are currently exploring a wider range of applications, including identifying liquids in intravenous therapy (IV), syringes, or drinks containing alcohol. Therefore, we envision great potential in enclosing a miniaturized NIRS scanner into a tool for tasks that require chemical information of objects in a Ubicomp context.

\section{Related Work}

Our work is rooted in related research focusing on the use of NIRS, light characteristics, and more general on the liquid identification of chemical compositions.

Near Infrared Spectroscopy

NIRS is a fast and non-destructive sensing technique which is conventionally used for composition analysis in chemistry. The whole process takes less than 10 seconds requiring only few samples, and can be directly performed in situ [12]. Scanning takes place by transmitting light beams with different wavelengths $(780 \mathrm{~nm} \sim 2500 \mathrm{~nm})$. At different wavelengths, variations of reflectance/absorbance by the chemical composition of the object result into a NIR spectrum. By analyzing this obtained spectrum, the scanned object's chemical information can be extracted.

NIRS has been successfully adopted in various industria fields for decades such as agriculture, chemistry and pharmacy [12]. A range of applications were built on top of this technique. For example, in agriculture NIRS was used for monitoring the quality of food, such as freshness of minced beef [13]. In medicine, NIRS has been widely adopted for non-invasive detection of human hemoglobin $(\mathrm{Hb})$ and other characteristics, thanks to its ability to penetrate bones and tissues [11]. 


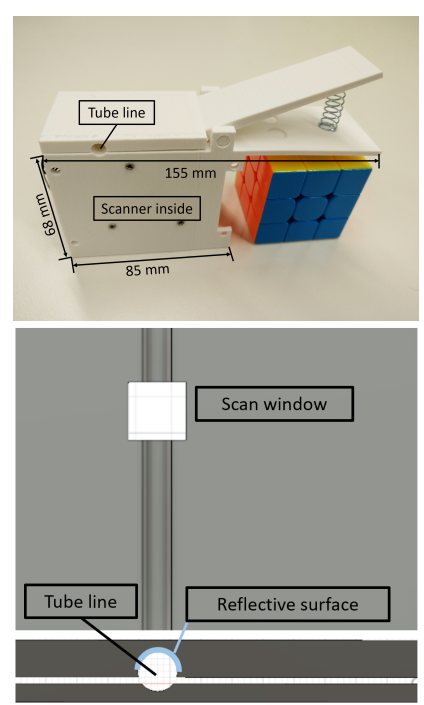

Figure 2: 3D printed clamp for miniaturized NIRS scanner (top), inner surface of clamp (middle) and side view (bottom)
Liquid Identification

Liquid identification includes both qualitative and quantitative analysis. The qualitative analysis aims to identify the category of liquids, while quantitative analysis attempts to quantify the concentration of compounds. In addition to NIRS, there are several alternative methods for identifying liquids. One of the most popular methods is the Drop Analysis Technology (DAT). The first DAT method, called Fiber Drop Analysis (FDA), was introduced by McMillan et al. in 1992 [14]. The authors identified liquids by studying intensity of lights that passed through the liquid drop pumped by a special head placed between two optical fibers. Subsequent studies of DAT used different sensing methods, including Capacitive Drop Analysis (CDA), Fiber-Capacitive Drop Analysis (FCDA), Image Drop Analysis (IDA) [14], and microwave sensing [6]. Nevertheless, DAT requires sampling using capillary tubes in a well-controlled experimental environment, whereas the NIRS technique can provide more detailed information in a timely manner in situ, and thus has important advantages over the DAT technique.

There are other liquid identification techniques that do not involve drop analysis. Compared to NIRS on a miniaturized scanner, most of the alternative methods provide limited advantages in liquid identification. For example, a refractometer is widely used in the agricultural sector to measure the sugar content in wines and juices. Yet it can only provide information on sugar concentration, and requires a small amount of samples $(<1 \mathrm{ml})$ to cover a sample well [4], which constrains its usage scenarios. Although graphene test papers can recognize a number of complex liquids with flexible shapes, it requires direct contac with the liquid [8]. Josephson Spectroscopy, similar to the NIRS technique, utilizes electromagnetic waves in the range of a few $\mathrm{GHz}$ to a few $\mathrm{THz}$, but is only able to provide coarse chemical information with a high Signal-to-Noise Ratio
(SNR) requirement [2]. An inkjet-printed Radio Frequency (RF) microstrip, introduced by Hassan et al., can only identify specific liquids such as water, ethanol, water/ethanol 50:50 mixture and synthetic engine oil [5]. In summary, most of the alternative techniques are rather bulky or costly, and are limited to a relatively small application niche. Our devised prototype addresses these shortcomings by exploring the state-of-the-art NIRS technique using a 3D printed clamp, providing a readily and rapidly liquid identification that can be used in wide deployment.

\section{Challenges in Identifying Liquids}

In addition to the shortcomings of identifying non-solid objects and especially liquids as pointed out by Klakegg et al. [10], the use of miniaturized NIRS scanners is challenging for non-professionals. These challenges include light scattering, caused by the flexibility of liquids, and lower received SNR due to low reflectance caused by the transparency of many liquids. It is worth noting that NIRS spectra are mostly complex with overlapped NIR reflectance or absorbance bands. Therefore, analyzing NIRS spectra with lower SNR introduces additional challenges in spectra data processing.

\section{Light Scattering}

Light scattering happens when the scanned surface is not perfectly flat. Scanning results can be affected by light scattering in different levels, while non-professionals lack knowledge to properly control light scattering for successful scanning [10]. Furthermore, since liquids take the shape of their container, and are susceptible to externa influence, light scattering may happen not only between two subsequent scans, but also during a single scan. This may result in a more dynamic interference compared to the scan of solid object. As the NIRS technique relies on spectrum analysis, light scattering can significantly affect the received signals, with a greater impact on the final analysis results. 


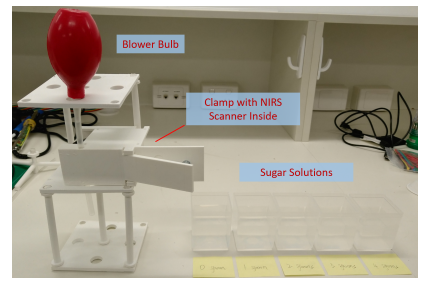

Figure 3: Experimental setup.

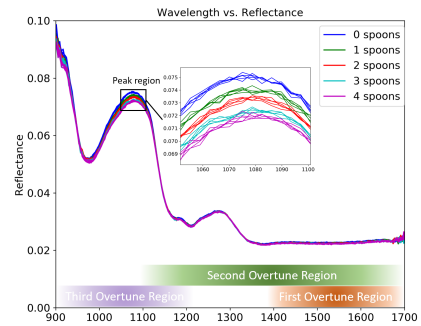

Figure 4: Raw NIRS data of sugar solutions with different concentrations.

\section{Low Reflectance}

NIRS spectra are acquired by receiving reflected lights after interacting with the scanned object. For those objects with low reflectance, the received light intensity can be very low, returning low SNR in the final NIR spectra. As most liquids are transparent, the majority of the light penetrates the liquids, while only a small portion of the light reflects back to the scanner to be detected, making liquids with similar chemical components hard to discriminate. As a consequence, in most cases of liquid identification, the transmission mode is adopted for the NIRS scanner, i.e. placing the receiver on the opposite of the transmitter, so that most of the light is received after interacting with the liquid. However, this configuration limits the placement of the NIRS scanner and, therefore, restricts the scenarios in which a scan can be performed.

\section{Design}

To address these challenges, we designed a 3D-printed clamp case for the NIRS scanner. As shown in Figure 2 with dimensions annotated, the clamp's size is about that of a Rubik's cube $(5.5 \mathrm{~cm}$ edge length). The tube is clamped to a tube line, which can then be scanned through the scan window in front of the tube. The scan window is covered by the clamp, blocking most of the ambient light from interfering with the NIRS scanner. The NIRS scanner is placed in the case right below the clamp, and controlled by an Android smartphone connected via Bluetooth.

It is worth noting that when inserted in the clamp case, the NIRS scanner works in reflectance rather than transmission mode for scanning liquids [12]. For increasing the SNR of the detected NIR signals, it is important that the clamp is printed with a white or high-reflectance material (we used white polylactic acid or PLA), since the surface on the opposite side of a scan window could act as a reflective surface. The reflective surface can reflect the NIR light beam emitted by the scanner, after passing through the tube and the liquid, which interacts again with the tube and the liquid before returning to the scan window for detection. Consequently, by lengthening the light path, the absorbance in specific wavelengths is increased. As the reflectance/absorbance spectrum acts as chemical fingerprint, the SNR in the final analyzed spectrum is also increased.

\section{Evaluation}

We experimentally evaluate our prototype by using our system to analyze sugar (common sucrose) levels in sugar-water solutions. We used five solutions with different levels of sugar concentration. The amount of water added as solvent remained constant (around $70 \mathrm{ml}$ ). We kept one solution as pure water for reference, while the other four solutions were mixed with one to four teaspoons of sugar (approximately four grams per spoon) respectively. The solution was pumped by a blower bulb and remained in a transparent tube attached to the blower bulb. The tube was clamped for scanning. For the experiment, we designed and 3D-printed a shelf as shown in Figure 3.

The scan was performed by the NIRS scanner connected to an Android smartphone via Bluetooth. Hadamard scan was adopted for higher SNR [12], with 228 evenly distributed measurement points in the $900 \mathrm{~nm} \sim 1700 \mathrm{~nm}$ wavelength span. Temperature and humidity were kept stable at room temperature, respectively $\sim 27$ degrees Celsius and $20 \%$. Each scan was taken only once with approximately two seconds of overall scanning time. In total, 25 scans were performed ( 5 scans per solution $\times 5$ solutions).

Results

Figure 4 shows the raw NIRS data for sugar solutions collected in the experiment. The overall reflectance is 

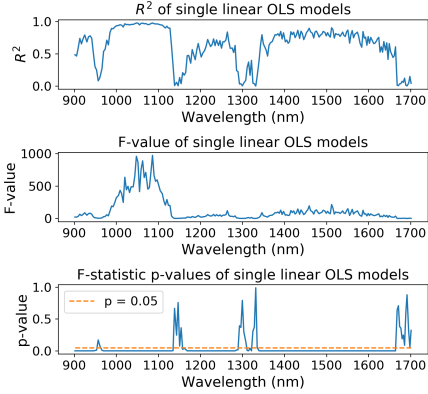

Figure 5: Wavelength evaluation by single linear OLS modeling.

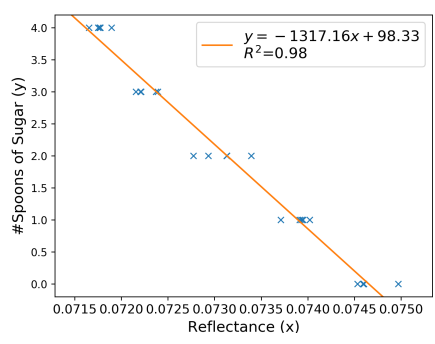

Figure 6: Single linear regression using OLS model. low $(<0.1)$, with most parts of the spectra overlapping with other measurements. A peak region is notable from around $1050 \mathrm{~nm}$ to $1100 \mathrm{~nm}$, where the differences of the reflectance are the most pronounced. The zoomed-in part of Figure 4 shows a clear monotone decrease as the sugar concentration of the sugar solution increases.

To further explore the most explanatory wavelengths for measuring sugar concentrations, we ran single linear ordinary least square (OLS) modeling to evaluate the significance of each wavelength. The objective was to predict sugar concentration (number of spoons of sugar) using the reflectance measurement data in a single wavelength. Thus, reflectance at each wavelength was considered as an independent variable in a single linear regression model. Figure 5 shows the statistical values for all the models. We observed that most of the wavelengths were statistically significant in the corresponding linear regression models $(p<0.05)$. We chose $1086 \mathrm{~nm}$ as the most explanatory wavelength since its model had both the largest $R^{2}$ value $\left(R^{2}=0.98\right)$ and F-value $(F=970.86)$. The fitting curve $(y=-1317.16 x+98.33)$ is shown in Figure 6

\section{Discussion and Conclusion}

We observed that the significant spectrum wavelengths can be separated in four spans: $900 \mathrm{~nm} \sim 953 \mathrm{~nm}, 965 \mathrm{~nm} \sim$ $1135 \mathrm{~nm}, 1158 \mathrm{~nm} \sim 1290 \mathrm{~nm}$, and $1340 \mathrm{~nm} \sim 1664 \mathrm{~nm}$. The four spans are due to the multiple overtune regions that originate when a single chemical compound interacts with the NIR lights. According to the NIRS theory, when a photon is absorbed by a molecule, it may transfer electrons from a low-energy to a high-energy molecule orbitals with different energy levels. The photon can only be absorbed if it contains the same energy as the gap between pre- and post-absorption. Since the photon energy is determined by its wavelength, and there are several energy gaps, multiple overtune regions are spread over the spectrum. We can further group the first two spans, the third span and the forth span as the third overtune region, the second overtune region and the first overtune region respectively, as shown in Figure 4.

This overtune phenomenon poses new challenges in liquid identification. Considering a solution with various solutes (chemical components), the characteristic wavelengths of these components may overlap at different overtune regions. Distinguishing liquids with similar chemical components will be challenging, given the fact that miniaturized NIRS scanners generally have lower resolution. In future work, we will explore ancillary methods to increase SNR including iterating the clamp modeling, signal pre-processing, etc.

In conclusion, we demonstrated the feasibility of our system to successfully identify five different concentrations of sugar-water solutions, which essentially adds new possible use cases of miniaturized NIRS scanner, such as identifying liquids in our everyday life. While previous work mainly focused on qualitative analysis (i.e., classification) [9], our system works for quantitative analysis as well. In our future work, we will address the challenges of turning the miniaturized NIRS scanner into a ubiquitous tool that could be used anywhere at any anytime, such as identifying liquids in hospitals (IV or syringe medicines), components of soft-drinks (check for allergy risks), or alcohol level detection. Potentially, this new tool could greatly benefit Ubicomp scientists, hobbyists, and those who require meaningful information about objects in their daily environment.

\section{Acknowledgement}

This work was supported by JST ERATO Grant Number JPMJER1501, Japan. 


\section{REFERENCES}

1. Amay J Bandodkar, Itthipon Jeerapan, and Joseph Wang. 2016. Wearable chemical sensors: Present challenges and future prospects. ACS Sensors 1, 5 (2016), 464-482.

2. Yuriy Divin, Matvey Lyatti, Ulrich Poppe, Knut Urban, and Valery Pavlovskiy. 2011. Josephson spectroscopy for identification of liquids. IEEE transactions on applied superconductivity 21, 3 (2011), 311-314.

3. Robert Geirhos, David HJ Janssen, Heiko H Schütt, Jonas Rauber, Matthias Bethge, and Felix A Wichmann. 2017. Comparing deep neural networks against humans: object recognition when the signal gets weaker. arXiv preprint arXiv:1706.06969 (2017).

4. Inc. Hanna Instruments. n.d. Digital Refractometer for Sugar Analysis in Wine, Must and Juice - HI96812 Hanna Instruments. https://goo.gl/y4jbrY. (n.d.). (Accessed on 07/12/2018).

5. Arshad Hassan, KiBae Lee, Jinho Bae, and Chong Hyun Lee. 2017. An inkjet-printed microstrip patch sensor for liquid identification. Sensors and Actuators A: Physical 268 (2017), 141-147.

6. Ming Huang, Jingjing Yang, Jiaqiang Wang, and Jinhui Peng. 2007. Microwave sensor for measuring the properties of a liquid drop. Measurement Science and Technology 18, 7 (2007), 1934

7. Texas Instruments Incorporated. n.d. DLP NIRscan Nano Evaluation Module.

http://www.ti.com/tool/DLPNIRNANOEVM. (n.d.) (Accessed on 07/11/2018).

8. Xin Jiang, Tingting Yang, Changli Li, Rujing Zhang, Li Zhang, Xuanliang Zhao, and Hongwei Zhu. 2017. Rapid Liquid Recognition and Quality Inspection with
Graphene Test Papers. Global Challenges 1, 6 (2017), 1700037.

9. Simon Klakegg, Jorge Goncalves, Chu Luo, Aku Visuri, Alexey Popov, Niels van Berkel, Zhanna Sarsenbayeva, Vassilis Kostakos, Simo Hosio, Scott Savage, and others. 2018. Assisted Medication Management in Elderly Care Using Miniaturised Near-Infrared Spectroscopy. Proceedings of the ACM on Interactive, Mobile, Wearable and Ubiquitous Technologies 2, 2 (2018), 69.

10. Simon Klakegg, Jorge Goncalves, Niels van Berkel, Chu Luo, Simo Hosio, and Vassilis Kostakos. 2017.

Towards Commoditised Near Infrared Spectroscopy. In Proceedings of the 2017 Conference on Designing Interactive Systems (DIS '17). ACM, New York, NY, USA, 515-527. DOI :

http://dx.doi.org/10.1145/3064663.3064738

11. Ai Liu, Gang Li, Zhigang Fu, Yang Guan, and Ling Lin 2018. Non-linearity correction in NIR absorption spectra by grouping modeling according to the content of analyte. Scientific reports 8, 1 (2018), 8564.

12. Metrohm NIRSystems. 2002. A guide to near-infrared spectroscopic analysis of industrial manufacturing processes. (2002)

13. Nicoletta Sinelli, Sara Limbo, Luisa Torri, Valentina Di Egidio, and Ernestina Casiraghi. 2010. Evaluation of freshness decay of minced beef stored in high-oxygen modified atmosphere packaged at different temperatures using NIR and MIR spectroscopy. Meat science 86, 3 (2010), 748-752.

14. Qing Song, GX Zhang, and ZR Qiu. 2005. Review of drop analysis technology for liquid property study. Opto-Electron. Rev. 13, 1 (2005), 1-8. 